\title{
Exosomal miR-451a Functions as a Tumor Suppressor in Hepatocellular Carcinoma by Targeting LPIN1
}

\author{
Shaorong Zhao Jianjun Li $^{\mathrm{a}}$ Guomin Zhang ${ }^{\mathrm{a}}$ Qiong Wang ${ }^{\mathrm{a}}$ Chao Wu \\ Quansheng Zhang $^{b}$ Hang Wang $^{a}$ Peiqing Sunc Rong Xianga \\ Shuang Yanga
}

aTianjin Key Laboratory of Tumor Microenvironment and Neurovascular Regulation, Medical College of Nankai University, Tianjin, China, ${ }^{\text {}}$ Tianjin Key Laboratory of Organ Transplantation, Tianjin First Center Hospital, Tianjin, China, 'Department of Cancer Biology, Wake Forest University School of Medicine, Winston-Salem, NC, USA

\section{Key Words}

Exosomes $\cdot \mathrm{miR}-451 \mathrm{a} \cdot$ Cell apoptosis $\cdot \mathrm{HCC} \cdot$ Angiogenesis

\begin{abstract}
Background/Aims: Emerging evidence suggests that exosomal microRNAs (miRNAs) mediate hepatoma progression through the post-translational regulation of their targets. However, characteristically-expressed miRNAs and their functions in the tumor and tumor-associated angiogenesis remain poorly understood. Methods: miRNA sequencing (HiSeq 2500 SE50) was performed to identify miRNA species that are involved in the hepatocellular carcinoma (HCC) pathogenesis. We identified miR-451a downregulation according to its expression and TCGA analysis. miR-451a was found to be mainly involved in cell viability, apoptosis, cell cycle and migration both in HCC and endothelial cell lines. LPIN1 was predicted to be a target of this miRNA based on TargetScan, GSEA analysis, and the Uniprot database. We performed real time PCR and dual luciferase assays to confirm these results. Results: We identified that miR-451a is significantly downregulated in serum-derived exosomes from HCC patients, as compared to expression in those from normal individuals. We further confirmed that overexpression of miR-451a functions in HCC and endothelia cells in vitro and in vivo. Exosomal miR-451a, as a tumor suppressor, was found to induce apoptosis both in HCC cell lines and human umbilical vein endothelial cells (HUVECs). In addition, miR-451a suppressed HUVEC migration, tube formation, and vascular permeability. Importantly, we demonstrated that LPIN1 is a critical target of miR-451a, and promotes apoptosis in both HCC and endothelial cells. Conclusion: Our study provides the novel finding that exosomal miR-451a targets LPIN1 to inhibit hepatocellular tumorigenesis by regulating tumor cell apoptosis and angiogenesis. These results have clinical implications regarding the deregulation of miRNAs in HCC.
\end{abstract}




\section{Cellular Physiology Cell Physiol Biochem 2019;53:19-35 \\ \begin{tabular}{l|l|l} 
DOI: 10.33594/000000118 & (c) 2019 The Author(s). Published by
\end{tabular} \\ \begin{tabular}{|l|l|l} 
Published online: 5 June 2019 & Cell Physiol Biochem Press GmbH\&Co. KG \\
\hline
\end{tabular} \\ Zhao et al.: Exosomal miR-451a Targets LPIN1 to Suppress HCC}

\section{Introduction}

Primary tumors of the liver represent the fifth most common type of cancer worldwide, and the third leading cause of cancer-related death [1]. Hepatocellular carcinoma (HCC) is the most frequent primary liver malignancy [2,3], and there are few effective biomarkers and techniques for the early diagnosis of this disease [4, 5]. Currently, alterations in exosomal cargo have been reported as biomarkers for a variety of human diseases including malignancies [6].

Exosomes are small membrane vesicles $(30-100 \mathrm{~nm}$ in diameter) that are derived from multivesicular bodies $[7,8]$. Circulating exosomes have been demonstrated to be highly stable and facilitate a new mode of cell-to-cell communication in serum and plasma; moreover, they are released by a variety of cells $[8,9]$. Exosomes, carrying proteins, lipids, and nucleic acids (DNA, mRNA, and miRNAs), have been explored for minimal molecular tracking, and more recently, serum exosomal microRNAs (miRNAs) have attracted attention as a potential source of novel HCC biomarkers [7, 9].

Recently, the role of miRNAs has become a major topic in cancer research [7]. miRNAs are small (17-21 nt), non-coding RNAs that can be delivered from HCC cells, via exosomes, and taken up by recipient cells including HCC cells themselves or other cells to regulate gene expression at the post-transcriptional level through the RNA interference pathway $[8,9]$. Previous studies have shown that the expression of multiple miRNAs, including circulating miRNAs, can be increased or decreased in HCC tumor tissues $[10,11]$. miRNAs are essential for the regulation of cancer cells, and are associated with cell viability (cell proliferation and apoptosis), metabolism, genome instability, tumor invasion, metastasis, angiogenesis, and immune responses [12]. For example, hepatic miR-291b-3p mediates glucose metabolism by upregulating PTEN expression, eventually resulting in the dysregulation of tumor cell growth $[13,14]$. Similarly, miR-34a functions as a tumor suppressor in breast cancer by inhibiting LDHA (lactate dehydrogenase A), which plays a critical role in tumor cell glucose metabolism [15]. However, miRNA profiles and their function remain largely obscure in HCC.

Therefore, we investigated the expression of circulating exosomal miRNAs in HCC patients and focused on one deregulated miRNA, namely miR-451a. Our results indicated that exosomal miR-451a promotes apoptosis in both HCC cells and endothelial cells. Consequently, ectopic expression of miR-451a perturbed HCC growth and tumor angiogenesis in vitro and in vivo. Collectively, our data identified a molecular mechanism through which exosomal miR-451a mediates HCC tumorigenesis, indicating that this molecule might be a potential target for HCC diagnosis.

\section{Materials and Methods}

\section{Clinical samples}

Serum samples from six HCC patients and two cohorts from the Tianjin First Central Hospital were subjected to miRNome sequencing. With the exception of eight samples, 71 samples, including 51 primary HCC tumor samples and 20 normal liver samples, were also obtained from Tianjin First Central Hospital, along with pathologic information. Serum samples were quickly frozen in liquid nitrogen. Surgically removed tissues were frozen until analysis or fixed and embedded.

\section{Exosome purification and electron microscopy}

Detailed protocols for preparing exosomes from serum and cell supernatant samples using the exosome isolation reagent (Ribobio Co., China) can be found in the manufacturer's protocol.

In addition, exosomes were isolated using ultracentrifugation. The serum was pre-cleared by centrifugation at $300 \times g$ for $5 \mathrm{~min}, 2000 \times g$ for $10 \mathrm{~min}$, and then at $10000 \times g$ for $60 \mathrm{~min}$. Exosomes were isolated by ultracentrifugation at $100000 \times \mathrm{g}$ for $130 \mathrm{~min}$, and washed with PBS using the same ultracentrifugation conditions. 


\section{Cellular Physiology Cell Physiol Biochem 2019;53:19-35 \begin{tabular}{l|l} 
DOI: 10.33594/000000118 & (c) 2019 The Author(s). Published by
\end{tabular} \\ \begin{tabular}{l|l} 
Published online: 5 June 2019 & Cell Physiol Biochem Press GmbH\&Co. KG \\
\hline
\end{tabular} \\ Zhao et al.: Exosomal miR-451a Targets LPIN1 to Suppress HCC}

For electron microscopy, exosomes were resuspended in $100 \mu \mathrm{l} \mathrm{PBS}$, fixed with $2 \%$ paraformaldehyde, loaded on 200-mesh Formvar-coated grids, contrasted, and embedded for imaging.

\section{Cell lines and reagents}

The human HCC cell lines Hep3B and HepG2 were obtained from ATCC. The HCC cell line SMMC-7721 and the normal human hepatocyte cell line L-02 were purchased from the Chinese Academy of Sciences (Shanghai, China). Hep3B and HepG2 cells were cultured in MEM containing 10\% fetal bovine serum (FBS), $100 \mathrm{U} / \mathrm{ml}$ penicillin/streptomycin, and $100 \mu \mathrm{g} / \mathrm{ml}$ non-essential amino acids. SMMC-7721 cells were grown in DMEM containing 10\% FBS and $100 \mathrm{U} / \mathrm{ml}$ penicillin/streptomycin. L-02 and human umbilical vein endothelial cells (HUVECs) were maintained in RPMI-1640 containing 10\% FBS and $100 \mathrm{U} / \mathrm{ml}$ penicillin/ streptomycin. miR-451a mimics and miR-451a mimic-Cy3 were purchased from Ribobio Co.

Cell viability, cell cycle distribution, and apoptosis assays

For cell viability assays, cells were seeded in 96-well plates in $100 \mu \mathrm{l}$ of medium, which was followed by transfection with different concentrations of miR-451a mimics. Six parallel wells were assigned for each group. At $0,24,48,72$, and $96 \mathrm{~h}$ after transfection, CCK-8 solution $(10 \mu \mathrm{l})$ was added to each well, and absorbance values were measured at $450 \mathrm{~nm}$ after incubation for $1.5 \mathrm{~h}$ at $37^{\circ} \mathrm{C}$.

Cell cycle analysis by flow cytometry was mainly based on the measurement of DNA content by staining with propidium iodide (PI, BD Biosciences, USA). Cells were collected, washed with PBS and then fixed in $3 \mathrm{ml}$ of $70 \%$ methanol at $-20{ }^{\circ} \mathrm{C}$. After $2 \mathrm{~h}$, cells were resuspended in $500 \mu \mathrm{l}$ PI staining solution at room temperature for $0.5 \mathrm{~h}$. Subsequently, cells were subjected to DNA fragmentation analysis using a FACScalibur (BD Biosciences). EdU assays were performed using the Cell-Light ${ }^{\mathrm{TM}}$ EdU Apollp®488 In vitro Imaging Kit (Ribobio Co.), following the manufacturer's protocol.

Quantification of apoptotic cells was performed by Annexin V-FITC/PI staining (BD Biosciences), in accordance with the manufacturer's instructions. TUNEL staining was performed according to a previous report [16].

\section{RNA extraction and quantitative RT-PCR}

Serum-derived exosomes were resuspended in $100 \mu \mathrm{l}$ PBS, quantified using BCA protein assay (Thermo, USA), lysed in Trizol reagent (Invitrogen, USA). Tissues were grinded using a homogenizer in Trizol reagent. Cells were collected, washed and lysed in Trizol reagent. Total RNA was isolated following the manufacturer's instructions.

RNA concentration was detected by BioDrop. Each reverse reaction was performed according to the EasyScript First-Strand cDNA Synthesis kit (Transgen, China).Quantitative PCR was performed using a standard SYBR-Green PCR kit protocol. Exosomes miR-451a and LPIN1 quantification were separately using U6 and GAPDH as internal reference. Primers were listed in the supplementary materials section (for all supplemental material see www.cellphysiolbiochem.com). GAPDH primers were described previously [17].

\section{Western blotting}

Western blotting was performed in accordance with a previous protocol [18]. Briefly, proteins were loaded on 5-12\% tris-acrylamide gels and membranes were blotted with specific antibodies. The LPIN1 antibody (Cat. 14906, Cell Signaling Technology, USA) was diluted to 1:1000. Bands were detected using a Gel imaging system (SYNGENE, USA).

\section{Plasmid construction and transfection}

SMMC-7721, Hep3B, and HUVECs (3 × $10^{5}$ cells/well) were seeded in 6-well plates, incubated overnight, and then transfected with miR-451a mimics (Ribobio Co., China) using Lipofectamine2000 (Invitrogen) and Opti-MEM (Corning) according to the manufacturer's instruction. Negative control (NC) is also purchased from Ribobio Co.

To construct the human LPIN1 overexpression vector, human LPIN1 cDNA was cloned from SMMC7721 cells and ligated into pLV-EF1 $\alpha$-MCS-IRES-Bsd (Biosettia Inc., USA). For gene silencing, shLPIN1 DNA oligomers were annealed and cloned into pLV-H1-EF1 $\alpha$-puro vector (Biosettia Inc., USA). These sequences are listed in the supplementary materials section. Scrambled control (SC) sequences were used as in a previous report [16]. 


\section{Cellular Physiology Cell Physiol Biochem 2019;53:19-35

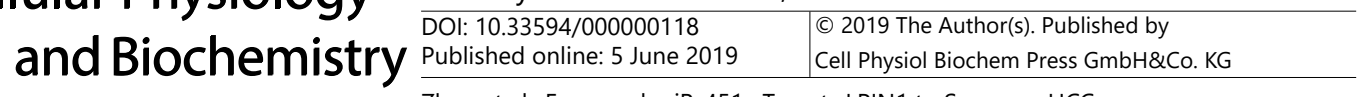 \\ Zhao et al.: Exosomal miR-451a Targets LPIN1 to Suppress HCC}

\section{Cell migration assay}

Cell migration was evaluated by performing wound-healing and transwell assays. For wound-healing assays, cells were seeded in 6 -well plates at $2 \times 10^{5}$ cells per well, and after $48 \mathrm{~h}$ transfection, the cell monolayer was scraped using a $10 \mu \mathrm{l}$ tip. The initial gap length and the residual gap length after wounding were calculated based on photomicrographs using ImageJ software.

For transwell assays, after transfection for $24 \mathrm{~h}$, cells were plated in 24 -well plates at $5 \times 10^{4}$ in the upper 8-nm chambers (BD bioscience). Medium containing 10\% FBS in the lower well and 2\% FBS in the upper chamber served as the chemoattractant. After another $16 \mathrm{~h}$ incubation, cells on the upper surface of the membrane were scraped off and those on the bottom were stained with crystal violet. These cells were photographed using an optical microscope and enumerated using Photoshop CS3.

\section{Tube formation and Matrigel plug assay}

A pre-chilled 48-well plate was coated with $150 \mu \mathrm{l}$ of Matrigel (BD bioscience) and incubated at $37^{\circ} \mathrm{C}$ for $30 \mathrm{~min}$. HUVECs $\left(3 \times 10^{4}\right.$ cells/well) were transfected with miR-451a and then seeded on this plate. After $5 \mathrm{~h}$, photographs were taken and the tubes were counted.

$\mathrm{BALB} / \mathrm{c}$ mice were administered a subcutaneous injection of $750 \mu \mathrm{l}$ of mixture containing $500 \mu \mathrm{l}$ Matrigel and $250 \mu \mathrm{l} \mathrm{EBM} 2$ medium with or without miR-451a. After 10 days, the Matrigel plugs were imaged and snap frozen in the presence of optimum cutting temperature (OCT) medium before sectioning. Frozen Matrigel sections $(5 \mu \mathrm{m})$ were fixed in cold methanol and immunostained with a CD31 antibody (ab28364, Abcam, USA).

\section{Immunofluorescence staining}

For this, $3 \times 10^{5}$ cells/well were seeded on cell chamber slides and placed in a 24-well plate. After transfection with miR-451a, cells were cultured for $24 \mathrm{~h}$ and fixed with $4 \%$ paraformaldehyde at room temperature for $15 \mathrm{~min}$. Slides were blocked with 2\% BSA and then incubated with an anti-ZO-1 antibody (Cat. 13663, Cell Signaling Technology) for $1 \mathrm{~h}$. After washing, slides were incubated with DAPI (Invitrogen) for 2 min. Images were obtained using a microscope (Olympus IX73).

\section{Dual luciferase reporter assay}

293T cells were cultured in 24-well plates at a density of $2 \times 10^{5}$ cells/well overnight, which was followed by co-transfection with miR-451a mimics, pmirGLO, and the pRL-TK plasmid. After $36 \mathrm{~h}$, the inhibition of miR-451a was quantified as the ratio of firefly luciferase activity to Renilla luciferase activity in each well.

\section{Tumor xenografts}

Male NOD/SCID mice (6-8-weeks-old) were separated randomly into two groups ( $\mathrm{n}=5$ each), and $2 \times$ $10^{6}$ SMMC-7721 cells were inoculated subcutaneously into each mouse, and tumors were allowed to form for 10 days. Tumors were peritumorally treated with miR-451a agomir or miRNA agomir negative control $(\mathrm{NC})$, and tumor volume $\left(\mathrm{mm}^{3}\right)$ was measured with calipers once every 3 days. All mouse experiments were conducted in accordance with the standard operating procedures approved by the Institute Research Ethics Committee at the Nankai University.

\section{Immunohistochemistry}

Immunohistochemistry staining was performed using paraffin-embedded human HCC tissues and mouse xenografts tumors. These tissues were probed separately with an antibody against LPIN1 (Cat. 14906, Cell Signaling Technology) and CD31 (ab28364, Abcam) at a 1:100 dilution. The expression of LPIN1 and CD31 was scored according to the percentage of positively-stained cells in the entire area.

\section{Statistical analysis}

Statistical analyses were performed using SPSS 23.0 software; the data from all experiments are presented as means $\pm S D$ and represent three independent experiments. A paired $t$-test was used to compare gene expression in tumor and adjacent non-tumor tissue samples. Where appropriate, a Student's $t$-test for unpaired observations was applied. A value of $P<0.05$ was considered significant. 


\begin{tabular}{|c|c|c|}
\hline \multirow{2}{*}{$\begin{array}{l}\text { Cellular Physiology } \\
\text { and Biochemistry }\end{array}$} & \multicolumn{2}{|c|}{ Cell Physiol Biochem 2019;53:19-35 } \\
\hline & $\begin{array}{l}\text { DOl: } 10.33594 / 000000118 \\
\text { Published online: } 5 \text { June } 2019\end{array}$ & $\begin{array}{l}\text { O } 2019 \text { The Author(s). Published by } \\
\text { Cell Physiol Biochem Press GmbH\&Co. KG }\end{array}$ \\
\hline
\end{tabular}

\section{Results}

\section{Differential miRNome in HCC serum-derived exosomes}

To identify exosomal miRNAs in human HCC, exosomes were purified from the sera of HCC patients and examined by electron microscopy. We observed that serum-derived exosomes exhibited a typical cup-shaped morphology (Fig. 1A). Meanwhile, serum exosomes were confirmed by the presence of three exosomal proteins, specifically CD63, Alix, and CD81 (Fig. 1B). Further, we applied miRNA sequencing (HiSeq 2500 SE50) to characterize miRNomes in the two cohorts, namely normal and patient serum samples. The abundance value of each known miRNA was normalized using "the number of reads per million (RPM) clean tags". We focused on downregulated miRNAs that might function as tumor suppressors in HCC. Results demonstrated that a total of 21 miRNAs (RPM $\geq 2000)$ were downregulated and differentially expressed in HCC patient serum-derived exosomes compared to expression in normal serum-derived exosomes (Supplementary Fig. S1A and Table 1). Among which, seven of them were significantly decreased $\geq 6$-fold (Table 1 and Fig. 1C). Additionally, TCGA database analysis verified that the expression of miR-26a-5p, miR-126-3p, miR-451a, miR92a-3p, and miR-191-5p was remarkably downregulated in HCC patients as compared to that in normal healthy individuals (Supplementary Fig. S1B).

Considering that the potential role of miR-451a in HCC tumorigenesis remains largely obscure, we further validated its expression in exosomes derived from normal and HCC patient sera. Our results confirmed that miR-451a expression was markedly reduced in HCC patient-derived serum exosomes (Fig. 1D). Additionally, downregulation of miR-451a in HCC tumor samples was observed in $64.7 \%$ (33/51) of clinical cases as compared expression in paired adjacent non-tumor tissues (Fig. 1E). Then we investigated the clinical significance of miR-451a in HCC. We divided all HCC patients into two groups (miR-451a low and miR-451a high) using the median level of miR-451a expression as a cutoff value. Clinical association analysis showed that the low level of miR-451a was correlated with high $\mathrm{T}$ classification and advanced clinical stages (Table 2). These results suggest an important role for exosomal miR-451a as a tumor suppressor in the regulation of HCC pathogenesis.

Fig. 1. miR-451a

is downregulated

in serum-derived

exosomes from

h e p a t o c e ll u la r

carcinoma (HCC)

patients. A, Electron microscope images of exosomes derived from HCC patient serum by ultracentrifugation (top) and isolation regent (bottom). B, Expression of molecular markers of exosomes, including CD63, Alix, and CD81, as
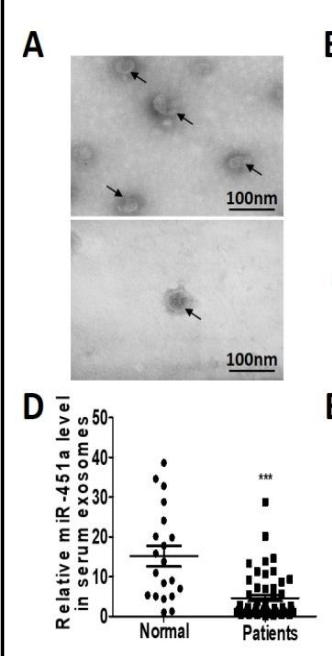

B

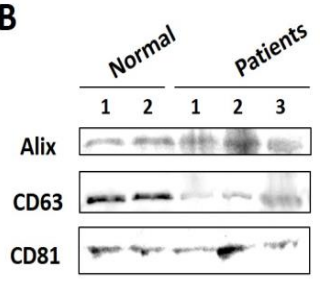

E

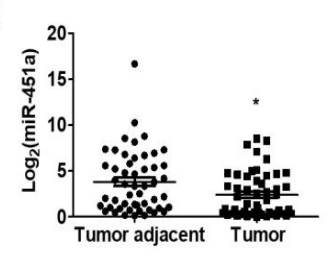

C

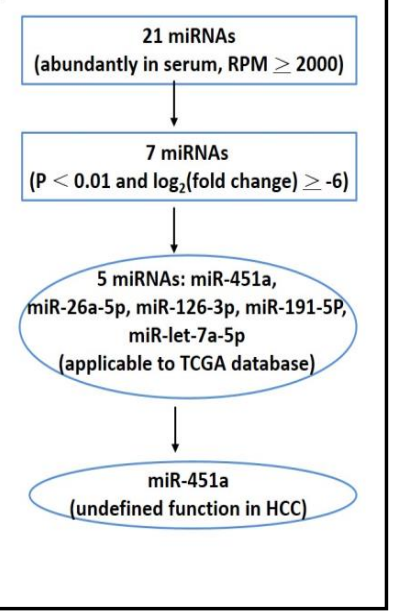
determined by western blotting. C, Screening strategy for differentially expressed exosomal miRNome in HCC patient serum. D, The expression level of exosomal miR-451a was assessed by quantitative PCR in normal and HCC serum. E, The expression level of miR-451a was assessed by quantitative PCR in HCC tissues and paired tumor adjacent tissues. ${ }^{*} \mathrm{P}<0.05,{ }^{* * *} \mathrm{P}<0.001$. 
Table 1. Deregulated miRNAs in serum-derived exosomes from hepatocellular carcinoma patients and normal individuals

\begin{tabular}{lccccc}
\hline miRNA_ID & Normal & Patients & Log $_{2}$ (fold-change) & P value & Significance \\
\hline hsa-miR-26a-5p & 12657.66 & 18.5662 & -9.4131 & $3.53 \mathrm{E}-09$ & $* *$ \\
hsa-miR-126-3p & 4506.747 & 9.1903 & -8.9378 & $2.24 \mathrm{E}-07$ & $* *$ \\
hsa-let-7a-5p & 5675.16 & 18.102 & -8.2923 & $3.47 \mathrm{E}-06$ & $* *$ \\
hsa-miR-451a & 29018.64 & 140.2674 & -7.6927 & $3.09 \mathrm{E}-05$ & $* *$ \\
hsa-miR-21-5p & 17346.43 & 106.1057 & -7.353 & 0.000199 & $* *$ \\
hsa-miR-92a-3p & 2812.607 & 18.2877 & -7.2649 & 0.000702 & $* *$ \\
hsa-miR-191-5p & 3088.834 & 24.8787 & -6.956 & 0.002331 & $* *$ \\
hsa-let-7b-5p & 2052.573 & 23.0221 & -6.4783 & 0.016819 & \\
hsa-miR-101-3p & 2267.633 & 29.2417 & -6.277 & 0.030935 & \\
hsa-let-7i-5p & 7532.777 & 112.6967 & -6.0627 & 0.05035 & \\
hsa-miR-185-5p & 5513.32 & 90.0459 & -5.9361 & 0.07711 & \\
hsa-miR-30d-5p & 3793.933 & 69.6231 & -5.768 & 0.131354 & \\
hsa-miR-320a & 11720.05 & 228.3639 & -5.6815 & 0.155584 & \\
hsa-miR-146a-5p & 9019.494 & 183.8051 & -5.6168 & 0.186673 & \\
hsa-miR-486-3p & 3368.1 & 103.4136 & -5.0254 & 0.668419 & \\
hsa-miR-22-3p & 17335.76 & 534.7057 & -5.0189 & 0.666535 & \\
hsa-miR-151a-3p & 2561.548 & 87.7252 & -4.8679 & 0.850311 & \\
hsa-miR-486-5p & 3060.315 & 163.6608 & -4.2249 & 0.444393 & $*$ \\
hsa-miR-148a-3p & 2780.815 & 683.6992 & -2.0241 & 0.000104 & $* *$ \\
hsa-miR-378a-3p & 2094.728 & 717.0255 & -1.5466 & $7.68 \mathrm{E}-066$ & $* *$ \\
hsa-miR-122-5p & 9506.105 & 3413.019 & -1.4778 & $4.83 \mathrm{E}-06$ & $* *$ \\
\hline
\end{tabular}

miR-451 a regulates HCC tumor cell apoptosis and migration in vitro

Next, we examined endogenous and exosomal miR451a expression in different HCC and normal hepatic cell lines. As shown in Fig. 2A, the expression of endogenous miR-451a was downregulated in a panel of three HCC cell lines including SMMC-7721, Hep3B, and HepG2 as compared to expression in the normal hepatic cell line $\mathrm{L}-02$. and Hep3B cell lines (Fig. 2B). These results demonstrated the suppression of miR-451a in endogenous HCC cells and in exosomes secreted into the tumor environment. We used SMMC-7721 and Hep3B cells for further research, as miR-451a expression was significantly decreased in these lines.

For this, we performed gain-of-function studies by transfecting miR-451a mimics into SMMC-7721 and Hep3B cells. CCK8 assays showed that miR-451a overexpression inhibited SMMC-7721 cell viability in a dose-dependent manner (Fig. 2C and Supplementary Fig. S2A). However, PI staining with subsequent FACS analysis demonstrated that overexpression of miR-451a did not alter cell cycle distribution in SMMC-7721 cells (Fig. S2B). Similarly, the number of miR-451a-transfected cells in the $S$ phase was unchanged based on an EdU staining assay (Supplementary Fig. S2C). Then, we determined whether miR-451a affects tumor cell apoptosis. FACS analysis after Annexin V staining and TUNEL assays demonstrated that tumor cell apoptosis was significantly increased in miR-451a-expressing SMMC-7721 cells (Fig. 2D and 2E). The same experiments were performed in Hep3B cells and similar results were obtained (Supplementary Fig. S3A-F), indicating that the growth-suppressive effect of miR-451a is due to increased apoptosis but not cell cycle arrest.

Moreover, ectopic expression of miR-451a resulted in a significant inhibition in cell migration in SMMC-7721 cells, based on wound-healing and transwell assays (Fig. 2F and 2G). Similar results were observed in Hep3B cells by performing transwell assays 


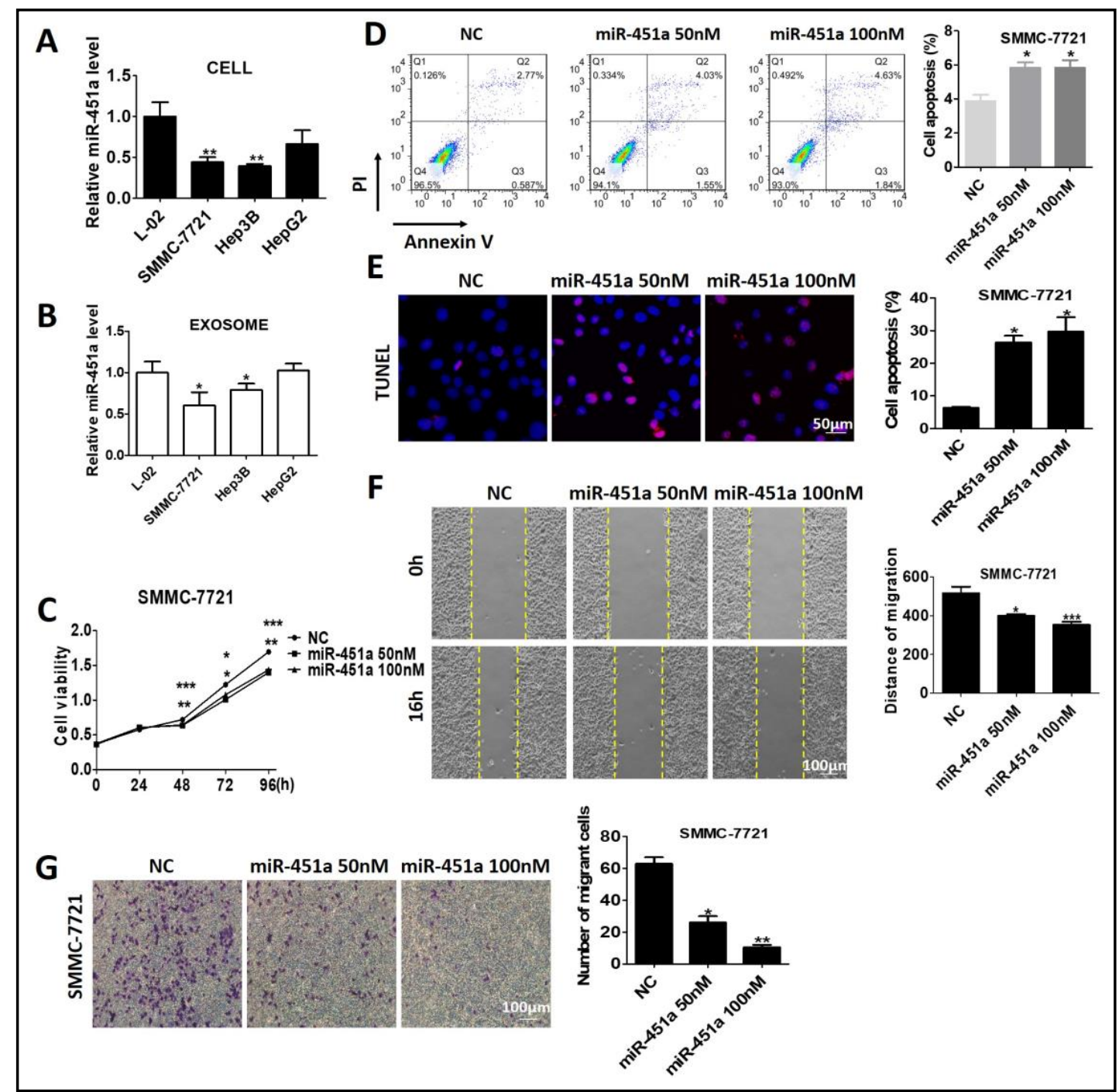

Fig. 2. miR-451a regulates hepatocellular carcinoma (HCC) cell apoptosis and migration. A and B, Cellular (A) and exosomal (B) RNA were extracted from different HCC and normal hepatic cell lines. The expression of miR-451a was examined by quantitative PCR. C, Different concentrations of miR-451a mimics were transfected into SMMC-7721 cells. Cell viability was measured by performing CCK8 assays. D and E, Different concentrations of miR-451a mimics were transfected into SMMC-7721 cells. Apoptosis was measured by Annexin V staining (D) and TUNEL (E) assays. PI, propidium iodide. F and G, Different concentrations of miR-451a mimics were transfected into SMMC-7721 cells. Cell migration was determined by wound-healing (F) and transwell (G) assays. NC, negative control; ${ }^{*} \mathrm{P}<0.05,{ }^{* *} \mathrm{P}<0.01,{ }^{* * *} \mathrm{P}<0.001$

(Supplementary Fig. S3G). These results collectively demonstrated a key role for miR-451a in the inhibition of HCC tumor cell growth and migration.

\section{Exosomal miR-451a significantly inhibits endothelial function in vitro}

Considering the previously identified role of miR-451a in suppressing AKT/mTOR pathway-mediated angiogenesis $[19,20]$, we evaluated the association between exosomal miR-451a and tumor angiogenesis in HCC. First, we investigated the exosomal delivery of miR-451a from the normal hepatocyte cell L-02 to human vein endothelium cells (HUVECs). The result showed that miR-451a expression of HUVECs was upregulated when cocultured with exosomes from L-02 cell lines, while it was reversed by GW4869, an inhibitor of exosome secretion (Supplementary Fig. S4A). Further, we detected the delivery of exosomal miR-451a 
from HCC cell lines to HUVECs. For this, we transfected SMMC-7721 cells with miR-451aCy3 mimics, and collected exosomes in the supernatant for co-culture with HUVECs. Results showed that after miR-451a overexpression in HCC cells, these molecules could be delivered to endothelial cells via exosomal secretion; however, this effect was significantly reduced in the presence of GW4869 (Fig. 3A). Quantitative PCR further revealed that overexpression of miR-451a in SMMC-7721 increased its endogenous expression and exosomal delivery to HUVECs (Supplementary Fig. S4B). Meanwhile, SMMC-7721-derived exosomal miR451a also inhibited HUVEC cell viability (Fig. 3B). Similar results were observed using Hep3B cells (Supplementary Fig. S4C-E). To explore the biological significance of miR-451a in endothelial function, we analyzed the effects of miR-451a overexpression on HUVEC proliferation, apoptosis, migration, tube formation, and permeability. Results showed that ectopic expression of miR-451a inhibited HUVEC viability by promoting apoptosis without affecting cell cycle distribution (Fig. 3C-E and Suppkementary Fig. S4F-G). Moreover, miR451a overexpression also inhibited HUVEC migration based on wound-healing and transwell assays (Fig. 4A-B). In addition, tube formation assays demonstrated that miR-451a inhibited this process in HUVECs in a dose-dependent manner (Fig. 4C). Previous studies reported that $\mathrm{ZO}-1$ localizes to intercellular tight junctions at the cytoplasmic membrane surface, and that this event can be assessed to indicate vessel permeability [21, 22]. Our immunofluorescence results illustrated that ectopic miR-451a resulted in cytomembrane localization of Z0-1, from the cytoplasm (Fig. 4D), implying that miR-451a might restrict vessel permeability. Notably, based on in vivo Matrigel plug assays, we demonstrated that microvessel density was decreased in mice treated with miR-451a agomir (Fig. 4E). Taken together, these results indicate that HCC-derived exosomal miR-451a inhibits tumor angiogenesis by disrupting endothelial functions such as apoptosis, migration, tube formation, and permeability.

\section{miR-451a targets LPIN1}

We further investigated the underlying mechanisms through which miR-451a regulates cell apoptosis and tumor angiogenesis. As miRNAs mainly function through the posttranscriptional inhibition of target genes, we thus sought to identify the potential targets of miR-451a during HCC pathogenesis. Interestingly, whole GO-based GSEA analysis revealed that miR-451a is associated with a variety of cell metabolic processes, and especially lipid catabolic-related activities (Supplementary Table S1). Moreover, we searched for candidate miR-451a target genes using publicly available databases including TargetScan (http:// www.targetscan.org), miRanda (http://www.microrna.org), and miRDB (http://www. mirdb.org). In total, 128 genes were identified as potential targets of miR-451a, among which 12 are associated with metabolic processes based on the Uniprot database (Fig. $5 \mathrm{~A}$ and Supplementary Table S2). Accordingly, quantitative PCR assays verified that the expression of LPIN1, AKR1B1, ATP5F1, GK, and ASAH1 was downregulated in miR-451aexpressing SMMC-7721 cells (Supplementary Fig. S5A). In addition, the expression of miR451a mimics resulted in significant repression of a luciferase reporter containing the 3 '-UTR of LPIN1, whereas those of AKR1B1, ATP5F1, GK, and ASAH1 did not respond to miR-451a overexpression (Fig. 5B and Supplementary Fig. S5B). We further constructed a reporter plasmid containing a mutant form of the LPIN1 3'-UTR (Fig. 5C). Luciferase assays revealed that whereas overexpression of miR-451a significantly inhibited reporter activity of the wild-type LPIN1 3'-UTR, this effect was totally abolished using the mutant LPIN 3'-UTR (Fig. 5D). Quantitative PCR and western blot assays further confirmed that overexpression of miR-451a decreased mRNA and protein levels of LPIN1 in both HCC and HUVEC cells (Fig. 5E-F and Supplementary Fig. 5C-F).

Moreover, the relationship between miR-451a and LPIN1 was demonstrated in HCC patients. We observed that LPIN1 expression was upregulated in tumor samples compared to that in adjacent non-tumor tissues (Fig. 5G). Pearson correlation coefficient analysis further identified a significant inverse relationship between miR-451a and LPIN1 (Fig. 5H). Previous studies reported a dual role for LPIN1 as an enzyme that catalyzing the dephosphorylation of phosphatidic acid as well as a co-transcriptional factor that regulates lipid homeostasis [23]. 


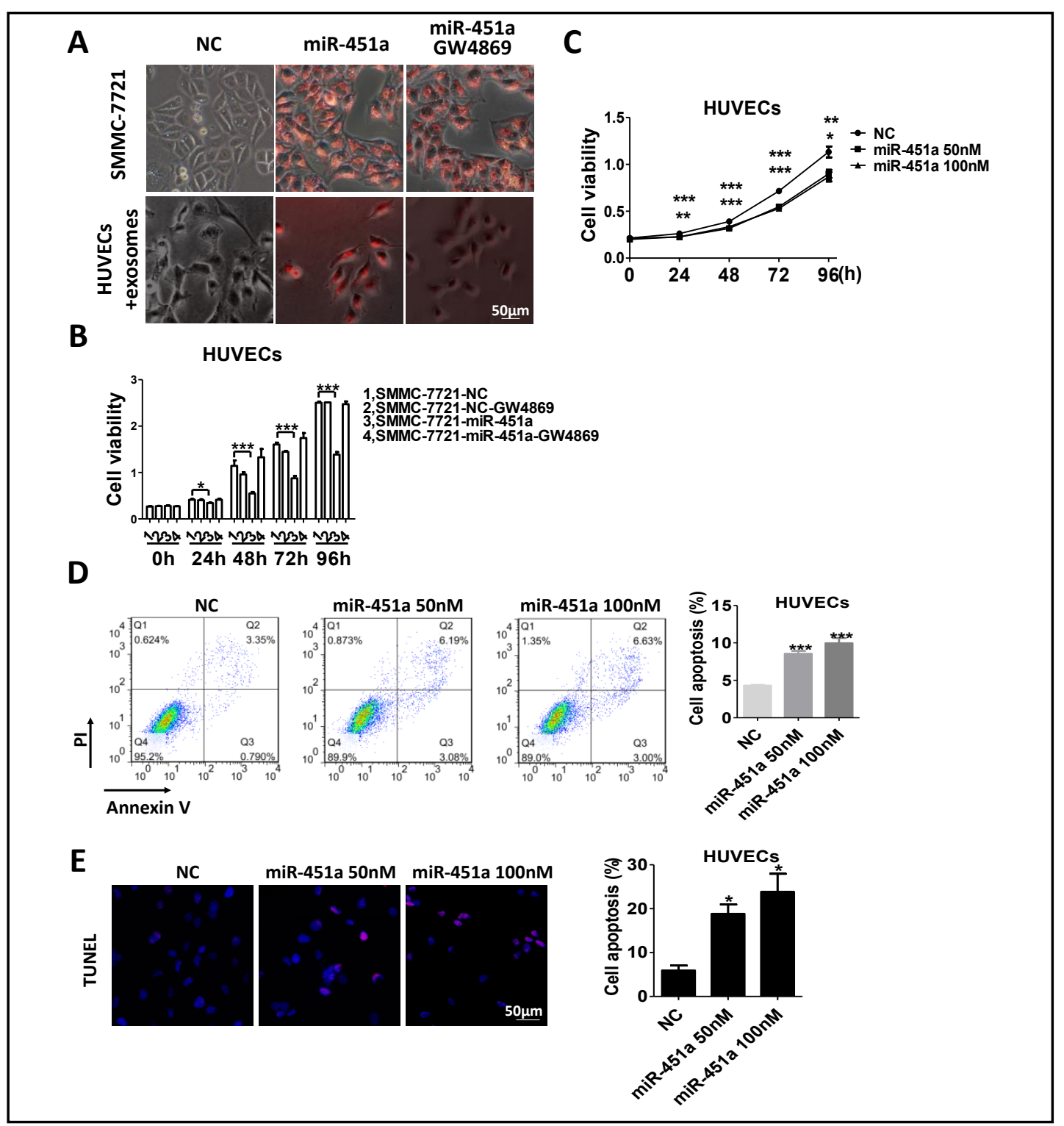

Fig. 3. Hepatocellular carcinoma (HCC)-derived exosomal miR-451a inhibits endothelial cell apoptosis. A, Exosomal miR-451a delivery from SMMC-7721 cells to human umbilical vein endothelial cells (HUVECs). Top: SMMC-7721 cells transfected with miR-451a-Cy3 mimics, and cultured in the presence or absence of exosome secretion inhibitor GW4869. Bottom: HUVECs incubated with SMMC-7721 exosomes. B, Exosomes were derived from SMMC-7721 cells transfected with miR-451a mimics in the presence or absence of GW4869, and were co-cultured with HUVECs. Cell viability was examined by CCK8 assays. C-E, Different concentrations of miR-451a mimics were transfected into HUVECs. Cell viability was measured by CCK8 assays (C). Apoptosis was measured by Annexin V (D) and TUNEL (E) assays. PI, propidium iodide; NC, negative control; ${ }^{*} \mathrm{P}<0.05,{ }^{* *} \mathrm{P}<0.01,{ }^{* * *} \mathrm{P}<0.001$.

Silenced LPIN1 was also shown to increase apoptosis in cancer cells [24, 25]. Taken together, LPIN1 might be function as an important target of miR-451a during the regulation of HCC growth and tumor angiogenesis, through the induction of apoptosis. 


\section{Cellular Physiology Cell Physiol Biochem 2019;53:19-35 \begin{tabular}{ll|l} 
and & $\begin{array}{l}\text { DOl: 10.33594/000000118 } \\
\text { a } 2019 \text { The Author(s). Published by } \\
\text { Cell Physiol Biochem Press GmbH\&Co. KG }\end{array}$ \\
\cline { 2 - 3 }
\end{tabular}

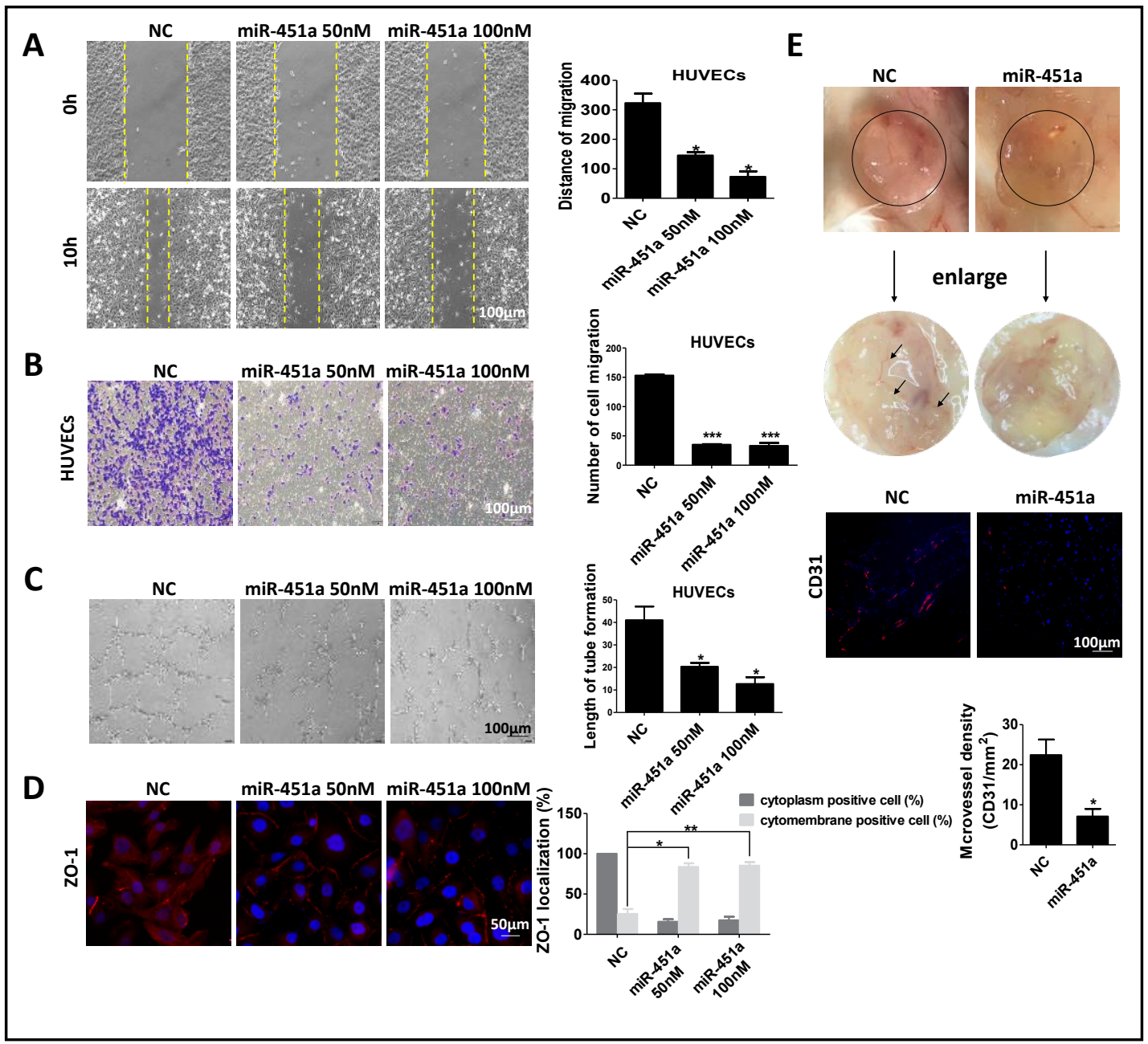

Fig. 4. miR-451a inhibits endothelial cell function. A-B, Different concentrations of miR-451a mimics were transfected into HUVECs. Cell migration was determined by wound-healing (A) and transwell (B) assays. C, HUVECs transfected with different concentrations of miR-451a mimics were seeded on a Matrigel layer. After $4 \mathrm{~h}$, tubular structures were manually counted. D, Representative images showing ZO-1 staining in HUVECs transfected with different concentrations of miR-451a mimics. ZO-1-positive staining in the cytoplasm or cytomembrane was quantified, and results are presented as the percentage of positive cells. E, Matrigel

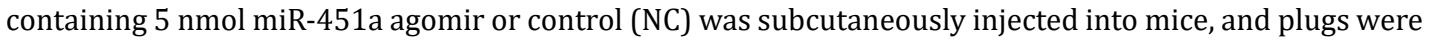
surgically removed after 10 days. The expression of CD31 in plugs was examined by immunofluorescent staining. NC, negative control; ${ }^{*} \mathrm{P}<0.05,{ }^{* *} \mathrm{P}<0.01,{ }^{* * *} \mathrm{P}<0.001$.

\section{miR-451a facilitates apoptosis mainly by targeting LPIN1}

We next investigated whether LPIN1, as a target of miR-451a, is critical for regulating HCC and endothelial cell apoptosis. For this, we overexpressed LPIN1 in control or miR-451aexpressing SMMC-7721 cells (Supplementary Fig. S6A). As shown in Fig. 6A, ectopic LPIN1 expression inhibited apoptosis in SMMC-7721 cells. Importantly, rescue of LPIN1 expression significantly attenuated miR-451a-induced SMMC-7721 apoptosis, implying that miR-451a induces SMMC-7721 cell apoptosis at least partially through the downregulation of LPIN1. We also knocked down LPIN1 expression in control or miR-451a-expressing SMMC-7721 cells (Supplementary Fig. S6B), which was followed by FACS analysis of Annexin V staining. Results showed that LPIN1 interference promoted SMMC-7721 cell apoptosis. However, co-transfection with miR-451a did not further augment apoptosis in LPIN1-knock down SMMC-7721 cells (Fig. 6B). This implies that LPIN1 might function downstream of miR-451a 


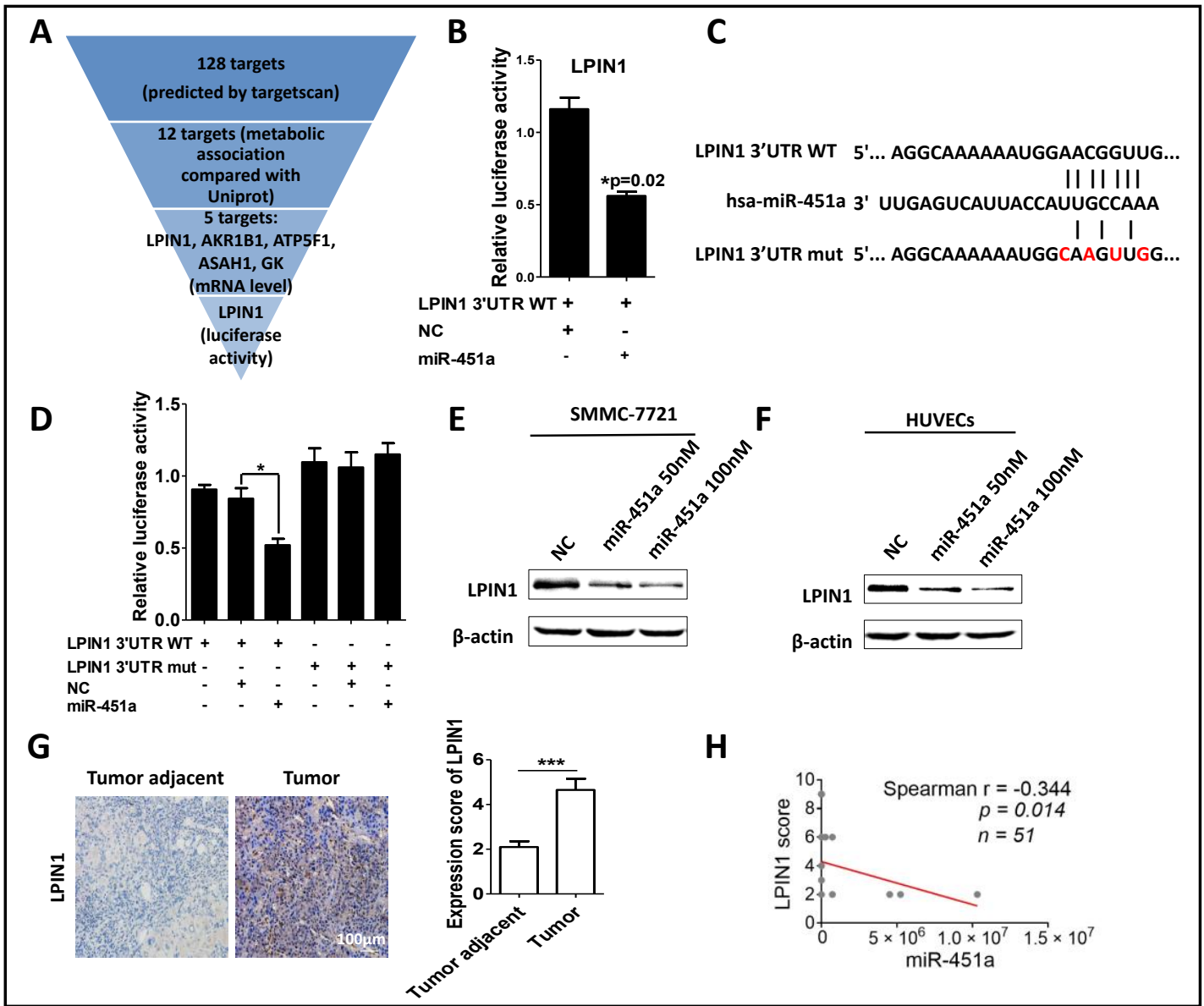

Fig. 5. LPIN1 is a target of miR-451a. A, Flow chart of miR-451a target screening. B, 293T cells were cotransfected with miR-451a mimics and wild-type promoter luciferase reporter constructs of LPIN1. Luciferase activities were determined $36 \mathrm{~h}$ after transfection. Luciferase values were normalized to Renilla activities. C, miR-451a binding site in the predicted target sequences of LPIN1 3'-UTR is indicated. D, 293T cells were transfected with wild-type or mutant LPIN1 promoter luciferase reporter constructs in the presence or absence of miR-451a mimics. Luciferase activities were determined $36 \mathrm{~h}$ after transfection. Luciferase values were normalized to Renilla activities. E and F, Different concentrations of miR-451a mimics were transfected into SMMC-7721 cells (E) and human umbilical vein endothelial cells (HUVECs) (F) for $48 \mathrm{~h}$. The expression of LPIN1 was detected by western blotting. G, The expression of LPIN1 was determined by immunohistochemistry (IHC) in tumor and adjacent non-tumor tissues from hepatocellular carcinoma (HCC) patients. H, Spearman's correlation analysis was performed to determine the relationship between miR-451a and LPIN1 expression in HCC patients. miR-451a levels were obtained from quantitative PCR and LPIN1 expression was based on IHC scores. NC, negative control; ${ }^{*} \mathrm{P}<0.05$, ${ }^{* * *} \mathrm{P}<0.001$.

signaling to mediate its effect on apoptosis. Similar results were observed in Hep3B cells and HUVECs (Supplementary Fig. S6C-H and 7A-B). These results demonstrated that LPIN1 is a critical target of miR-451a during the regulation of HCC and endothelial cell apoptosis.

miR-451a regulates tumor cell apoptosis and angiogenesis in HCC xenografts in vivo

To further determine the role of miR-451a in HCC progression in vivo, we performed tumor xenograft studies using NOD/SCID mice. SMMC-7721 cells were subcutaneously transplanted into NOD/SCID mice (Fig. 8A). Tumor development was allowed to occur for 10 days, which was followed by peritumoral treatment with $5 \mathrm{nmol}$ miR-451a agomir (once every 3 days) for another 15 days. Results indicated that the addition of miR-451a agomir effectively inhibited tumor growth in NOD/SCID mice, with a corresponding decrease in 


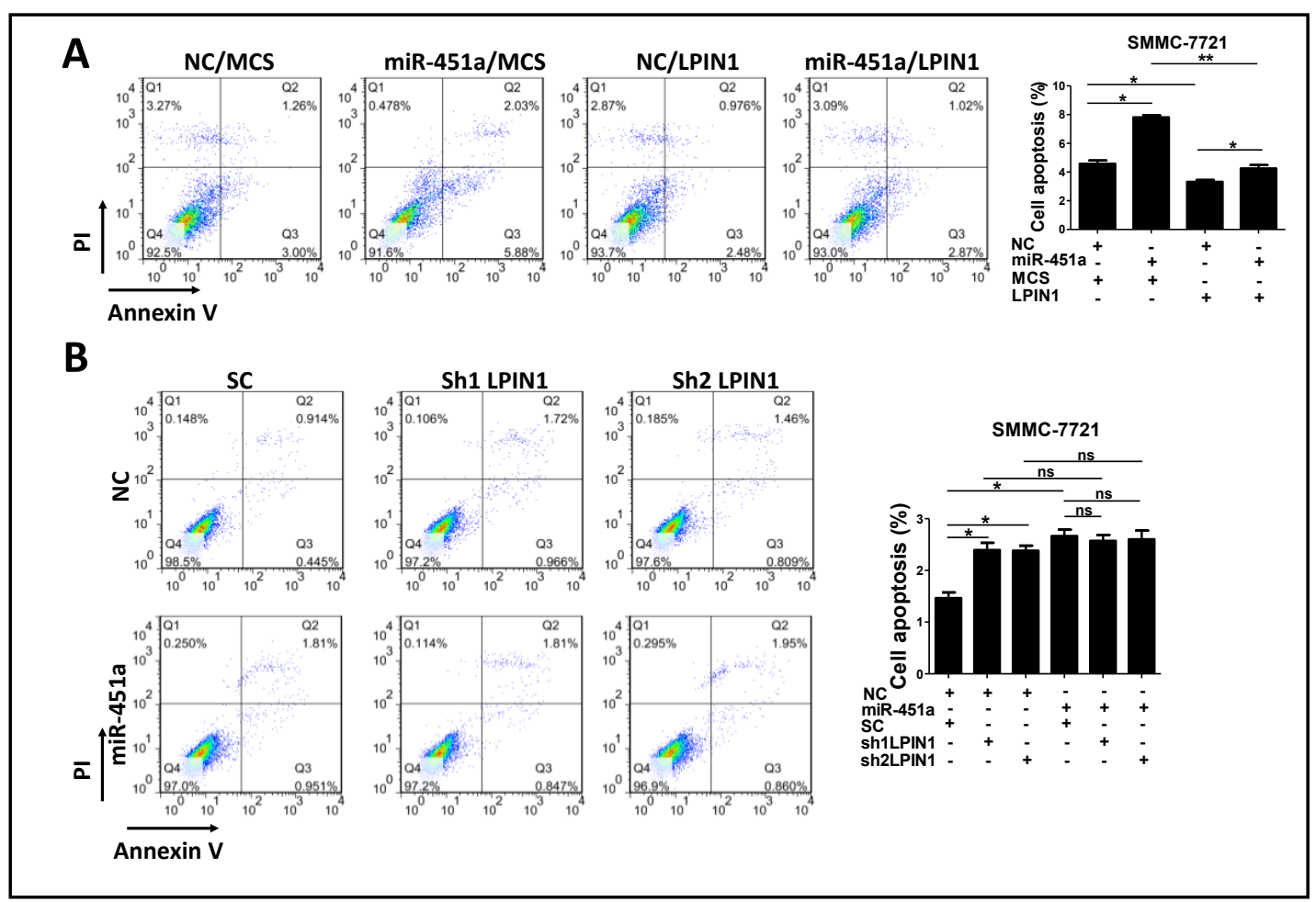

Fig. 6. LPIN1 is a critical target during miR-451a-induced SMMC-7721 cell apoptosis. A, SMMC-7721 cells were transfected with an LPIN1 expression plasmid in the presence or absence of miR-451a mimics. Apoptosis was determined by Annexin V assays. B, SMMC-7721 cells were transfected with specific LPIN1targeting shRNAs in the presence or absence of miR-451a mimics. Apoptosis was determined by Annexin $\mathrm{V}$ assays. ns, not significant; $\mathrm{NC}$, negative control; $\mathrm{SC}$, shRNA control; ${ }^{*} \mathrm{P}<0.05$, ${ }^{* *} \mathrm{P}<0.01$.

tumor weight and volume of greater than $50 \%$, as compared to those in control mice (Fig. 8B-D). Moreover, no adverse effects on other gross measures such as body weight or toxic death were observed in miR-451a agomir-treated mice (Fig. 8E). Quantitative PCR, western blotting, and immunohistochemistry further confirmed that LPIN1 was significantly downregulated in miR-451a agomir-treated tumors, demonstrating an inverse relationship between miR-451a and LPIN1 expression in HCC xenografts in vivo (Fig. 8F-H). Importantly, TUNEL assays demonstrated an increased proportion of apoptotic cells in tumors from miR-451a agomir-treated mice as compared to that in tumors from control mice (Fig. 8I). However, immunohistochemistry staining for CD31 revealed that tumor microvessel density decreased in miR-451a agomir-treated mice (Fig. 8J). These results collectively demonstrate that elevated miR-451a expression leads to inhibition of HCC growth and tumor angiogenesis via apoptosis in vivo.

\section{Discussion}

HCC has become one of the most prevalent causes of cancer-related death [26], and thus there is an urgent need for novel effective diagnostic approaches and therapies. Serumderived exosomes undoubtedly represent a convenient target for diagnosis. Previous studies have reported that exosomes exhibit superior ability to deliver cargos to suppress tumor growth, especially compared to that of liposomes [27]. In our study, we identified that miR-451a is significantly downregulated in serum-derived exosomes from HCC patients. Moreover, exosomal miR-451a was found to function as a tumor suppressor by inducing HCC and endothelial cell apoptosis. Ectopic expression of miR-451a significantly impaired 


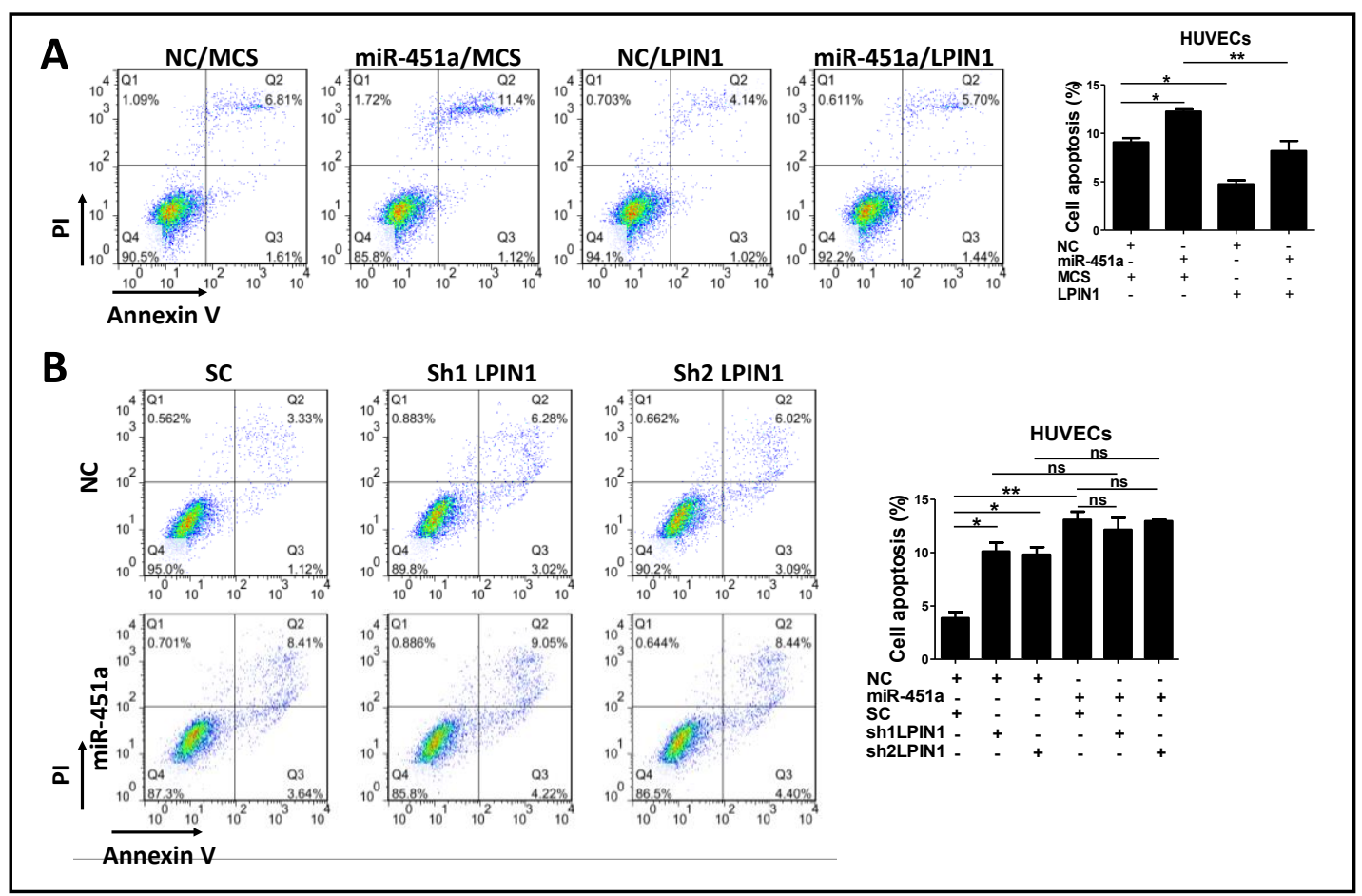

Fig. 7. LPIN1 is a critical target during miR-451a-induced HUVEC cell apoptosis. A, Human umbilical vein endothelial cells (HUVECs) were transfected with an LPIN1 expression plasmid in the presence or absence of miR-451a mimics. Apoptosis was determined by Annexin V assays. B, HUVECs were transfected with specific LPIN1-targeting shRNAs in the presence or absence of miR-451a mimics. Apoptosis was determined by Annexin V assays. ns, not significant; NC, negative control; $\mathrm{SC}$, shRNA control; ${ }^{*} \mathrm{P}<0.05,{ }^{* *} \mathrm{P}<0.01$.

HCC growth and tumor angiogenesis in vitro and in vivo. Thus, exosomal miR-451a could be applied as a biomarker for the serum-based diagnosis of and as a therapeutic target for HCC.

Previous reports have shown that miR-451a is downregulated in many types of human cancers $[19,28,29]$, which is consistent with our results demonstrating that miR-451a is a tumor suppressor for HCC. Additionally, miR-451a was found to be associated with various pathophysiological functions. For example, downregulated miR-451a is associated with large tumor diameter, poor prognosis based on pathological stage, and lymph node metastasis in lung cancer [30]. In contrast, ectopic miR-451a was found to decrease 14-3-33 expression and in turn increase ER $\alpha$ expression, resulting in apoptosis and reduced p-AKT and p-mTOR activity in breast cancer cells [31]. In addition, a role for miR-451a in HCC cell proliferation, migration, and angiogenesis has been demonstrated. Our study mostly focused on the role of exosomal miR-451a, and the results are similar with those of previous studies. Li et al. reported that miR-451a upregulation leads to G0/G1 cell cycle arrest in HCC cells [32]. In our study, we found that miR-451a induces G0/G1 arrest only in Hep3B cells treated with $100 \mathrm{nM}$. These results imply that miR-451a functions differently in cell cycle distribution in different cell types. Our results further confirmed those of a previous study regarding the role of miR451a in tumor-associated angiogenesis $[31,33]$. We observed that miR-451a dysfunction is involved in tube formation and vessel permeability in HCC-associated endothelial cells, thus leading to impaired tumor angiogenesis. Moreover, we observed a possible correlation between miR-451a and lipid homeostasis during HCC progression, which requires further research.

LPIN1 plays a role in lipid metabolism in various tissues and cell types. The inhibition of lipogenesis was previously reported to affect cancer cellular processes including apoptosis, migration. He et al. found that LPIN1 silencing increases apoptosis in basal-like triplenegative breast cancer [25]. Our study confirmed that miR-451a targeted LPIN1 regulated cell 


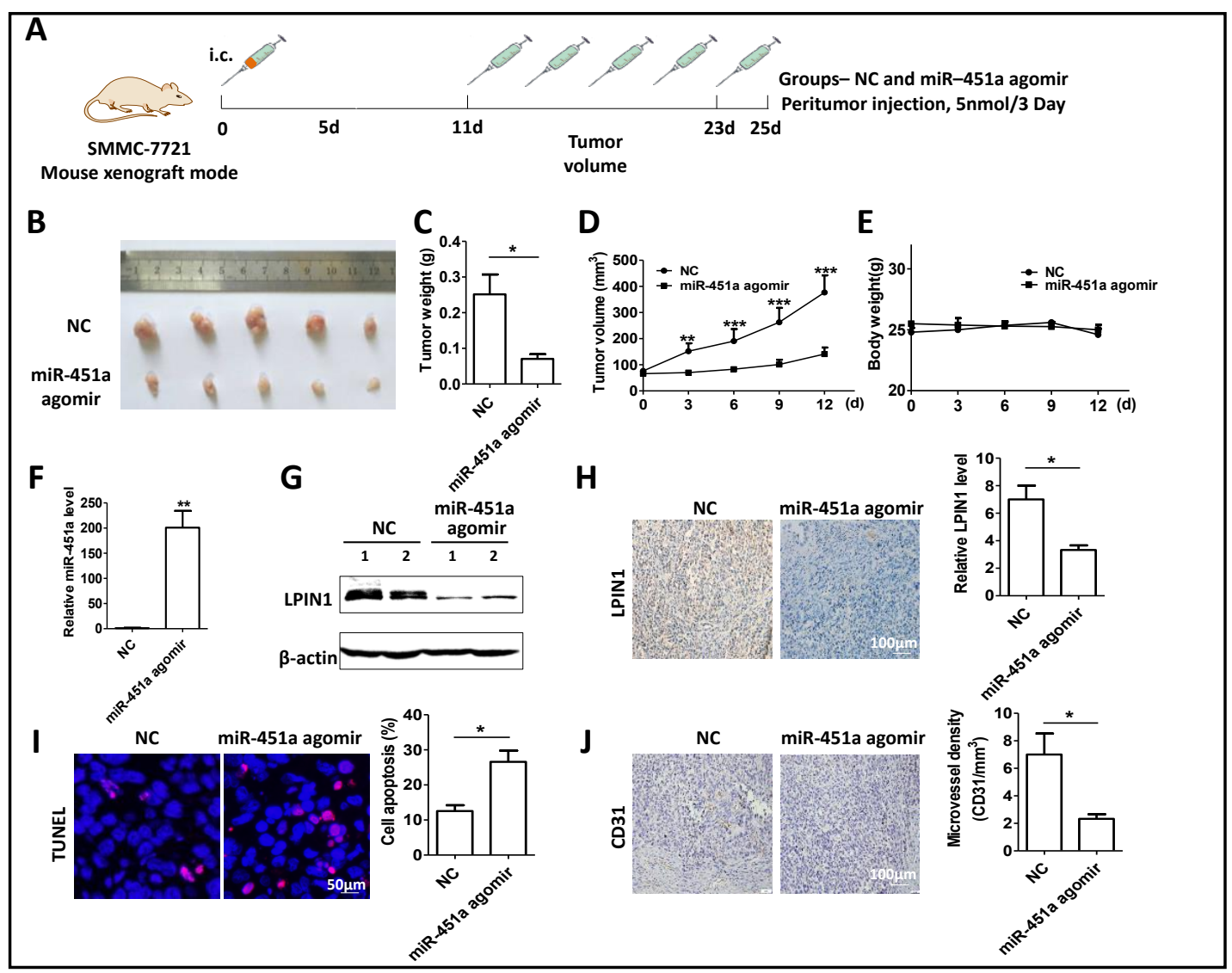

Fig. 8. Elevated expression of miR-451a inhibits hepatocellular carcinoma (HCC) tumor growth and angiogenesis in vivo. A, SMMC-7721 cells were subcutaneously injected into male NOD/SCID mice $(n=5)$. Tumor development was allowed to occur for 10 days, and then mice were peritumorally treated with miR451a agomir or negative control (NC) (once every three days) for another 15 days. B, Tumors from miR451a agomir- and NC-treated mice are shown. C-E, Approximate tumor weight (C), tumor volume (D), and body weight (E) of mice were measured. F, Expression of miR-451a in HCC xenografts was examined by quantitative PCR. G and H, The expression of LPIN1 in HCC xenografts was examined by western blotting (G) and immunohistochemical (IHC) staining (H). I and J, Apoptosis in HCC xenografts was assessed by TUNEL assays (I) and IHC staining for CD31 (J). ${ }^{*} \mathrm{P}<0.05,{ }^{* *} \mathrm{P}<0.01,{ }^{* * *} \mathrm{P}<0.001$.

apoptosis. Moreover, LPIN1 depletion was also reported to decrease prostate cell migration [34]. That research indicated that the regulation of migration by miR-451a could also be mediated by LPIN1. In addition, miR-451a functions mainly through the inhibition of target genes that are associated with intracellular signaling pathways. Thus, other target genes and signaling pathways are also suggested to regulate the growth of tumor cells, and therefore, the role of other potential metabolically-unrelated target genes and signaling pathways in the growth of hepatoma cells requires further investigation. In our study, we demonstrated that miR-451a is closely related to lipid metabolism through its LPIN1-targeting function.

Herein, the function of miR-451a was found to be related to its ability to target LPIN1, a factor that regulates lipid homeostasis, implying the molecular mechanism through which miR-451a regulates HCC pathogenesis. Importantly, the antitumor effects of miR-451a, such as the inhibition of tumor growth and angiogenesis, were identified both in vitro and in vivo. Taken together with the previously established clinical roles of miRNAs [10, 27], we provide functional evidence that miR-451a might be a potential target for HCC diagnosis and gene therapy. 


\section{Cellular Physiology Cell Physiol Biochem 2019;53:19-35

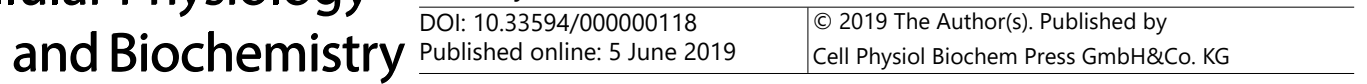 \\ Zhao et al.: Exosomal miR-451a Targets LPIN1 to Suppress HCC}

\section{Conclusion}

Collectively, we demonstrated that miR-451a is downregulated in serum exosomes derived from HCC patients, implying that exosomal miR-451a is a potential target for HCC diagnosis. Ectopic expression of miR-451a perturbed HCC growth and tumor angiogenesis via apoptosis in vitro and in vivo. Moreover, LPIN1, a lipid metabolism associated factor, was found to be a critical target of miR-451a in HCC and endothelial cells. Our study provides new insight into the mechanisms of hepatocellular carcinoma. However, further studies on feasibility are still required before miR-451a can be targeted for clinical applications.

\section{Acknowledgements}

This work was supported by grants from the International S\&T Cooperation Program of China (No. 2015DFA30420), the National Natural Science Foundation of China (No. 81472545), and the Natural Science Foundation of Tianjin City (No. 17JCZDJC36600). The study was approved by the institutional ethics committees at the Tianjin First Central Hospital and Medical School of Nankai University. All patients provided informed consent according to the latest version of the Helsinki Declaration on human research ethics.

\section{Disclosure Statement}

All authors declare that they have no competing interests.

\section{References}

1 Ramadori P, Cubero FJ, Liedtke C, Trautwein C, Nevzorova YA: Alcohol and Hepatocellular Carcinoma: Adding Fuel to the Flame. Cancers 2017;9:130.

2 Siegel RL, Miller KD, Jemal A: Cancer Statistics, 2017. CA Cancer J Clin 2017;67:7-30.

3 Fornari F, Ferracin M, Trere D, Milazzo M, Marinelli S, Galassi M, Venerandi L, Pollutri D, Patrizi C, Borghi A, Fosci FG, Stefanini GF, Negrini M, Bolondi L, Gramantieri L: Circulating microRNAs, miR-939, miR-595, miR519d and miR-494, Identify cirrhotic patients with HCC. PloS One 2015;10:e0141448.

4 Nicoud IB, Jones CM, Pierce JM, Earl TM, Matrisian LM, Chari RS, Gorden DL: Warm hepatic ischemiareperfusion promotes growth of colorectal carcinoma micrometastases in mouse liver via matrix metalloproteinase-9 induction. Cancer Res 2007;67:2720-2728.

5 Bolton JS, O’Connell MJ, Mahoney MR, Farr GH, Jr., Fitch TR, Maples WJ, Nagorney DM, Rubin J, Fuloria J, Steen PD, Alberts SR: Hepatic arterial infusion and systemic chemotherapy after multiple metastasectomy in patients with colorectal carcinoma metastatic to the liver: A north central cancer treatment group (NCCTG) phase II study, 92-46-52. Clin Colorectal Cancer 2012;11:31-37.

6 Benhamou Y, Di Martino V, Bochet M, Colombet G, Thibault V, Liou A, Katlama C, Poynard T, Multivir CG: Factors affecting liver fibrosis in human immunodeficiency virus-and hepatitis c virus-coinfected patients: Impact of protease inhibitor therapy. Hepatology 2001;34:283-287.

7 Sohn W, Kim J, Kang SH, Yang SR, Cho JY, Cho HC, Shim SG, Paik YH: Serum exosomal microRNAs as novel biomarkers for hepatocellular carcinoma. Exp Mol Med 2015;47:e184.

8 Sugimachi K, Matsumura T, Hirata H, Uchi R, Ueda M, Ueo H, Shinden Y, Iguchi T, Eguchi H, Shirabe K, Ochiya T, Maehara Y, Mimori K: Identification of a bona fide microRNA biomarker in serum exosomes that predicts hepatocellular carcinoma recurrence after liver transplantation. Br J Cancer 2015;112:532-538.

9 Tomasetti M, Lee W, Santarelli L, Neuzil J: Exosome-derived microRNAs in cancer metabolism: Possible implications in cancer diagnostics and therapy. Exp Mol Med 2017;49:e285.

10 Borel F, Konstantinova P, Jansen PL: Diagnostic and therapeutic potential of miRNA signatures in patients with hepatocellular carcinoma. J Hepatol 2012;56:1371-1383. 


\section{Cellular Physiology Cell Physiol Biochem 2019;53:19-35 \begin{tabular}{l|l|l}
\hline DOI: $10.33594 / 000000118$ & 2019 The Author(s). Published by
\end{tabular} \\ \begin{tabular}{l|l} 
Published online: 5 June 2019 & Cell Physiol Biochem Press GmbH\&Co. KG \\
\hline
\end{tabular} \\ Zhao et al.: Exosomal miR-451a Targets LPIN1 to Suppress HCC}

11 Giordano S, Columbano A: MicroRNAs: New tools for diagnosis, prognosis, and therapy in hepatocellular carcinoma? Hepatology 2013;57:840-847.

12 Ruan K, Fang X, Ouyang G: MicroRNAs: Novel regulators in the hallmarks of human cancer. Cancer lett 2009;285:116-126.

13 Guo J, Dou L, Meng X, Chen Z, Yang W, Fang W, Yang C, Huang X, Tang W, Yang J, Li J: Hepatic miR-291b3 p mediated glucose metabolism by directly targeting p65 to upregulate PTEN expression. Sci Rep 2017;7:39899.

14 Kim S, Domon-Dell C, Kang J, Chung DH, Freund JN, Evers BM: Down-regulation of the tumor suppressor PTEN by the tumor necrosis factor-alpha/nuclear factor-kappaB (NF-kappaB)-inducing kinase/NF-kappaB pathway is linked to a default IkappaB-alpha autoregulatory loop. J Bio Chem 2004;279:4285-4291.

15 Xiao X, Huang X, Ye F, Chen B, Song C, Wen J, Zhang Z, Zheng G, Tang H, Xie X: The miR-34a-LDHA axis regulates glucose metabolism and tumor growth in breast cancer. Sci Rep 2016;6:21735.

16 Shen W, Chang A, Wang J, Zhou W, Gao R, Li J, Xu Y, Luo X, Xiang R, Luo N, Stupack DG: TIFA, an inflammatory signaling adaptor, is tumor suppressive for liver cancer. Oncogenesis 2015;4:e173.

17 Zhang J, Zhou C, Jiang H, Liang L, Shi W, Zhang Q Sun P, Xiang R, Wang Y, Yang S: Zeb1 induces ERalpha promoter hypermethylation and confers antiestrogen resistance in breast cancer. Cell Death Dis 2017;8:e2732.

18 Xu Y, Dong X, Qi P, Ye Y, Shen W, Leng L, Wang L, Li X, Luo X, Chen Y, Sun P, Xiang R, Li N: Sox2 communicates with Tregs through CCL1 to promote the stemness property of breast cancer cells. Stem cells 2017;35:2351-2365.

19 Riquelme I, Tapia O, Leal P, Sandoval A, Varga MG, Letelier P, Buchegger K, Bizama C, Espinoza JA, Peek RM, Araya JC, Roa JC: miR-101-2, miR-125b-2 and miR-451a act as potential tumor suppressors in gastric cancer through regulation of the PI3K/AKT/mTOR pathway. Cell Oncol (Dordr) 2016;39:23-33.

20 Yadav L, Puri N, Rastogi V, Satpute P, Sharma V: Tumour angiogenesis and angiogenic inhibitors: A review. J Clin Diagn Res 2015;9:XE01-XE05.

21 Xu J, Liu Z, Zhan W, Jiang R, Yang C, Zhan H, Xiong Y: Recombinant TsP53 modulates intestinal epithelial barrier integrity via upregulation of ZO-1 in LPS induced septic mice. Mol Med Rep 2017;17:1212-1218.

22 Ke J, Shao W, Jiang Y, Xu J, Li F, Qin J: MicroRNA103 regulates tumorigenesis in colorectal cancer by targeting ZO-1 Mol Med Rep 2018;17:783-788.

23 Naderi M, Pazouki A, Arefian E, Hashemi SM, Jamshidi-Adegani F, Gholamalamdari O, Soudi S, Azadmanesh K, Mirab Samiee S, Merat S, Gholami Fesharaki M, Mondanizadeh M, Vasei M, Soleimani M: Two triacylglycerol pathway genes, CTDNEP1 and LPIN1, are down-regulated by hsa-miR-122-5p in hepatocytes. Arch Iran Med 2017;20:165-171.

24 Brohee L DS, Willems J, Arnould T, Colige AC, Deroanne CF: Lipin-1 regulates cancer cell phenotype and is a potential target to potentiate rapamycin treatment. Oncotarget 2015;10:11264-11280.

25 He J, Zhang F, Tay LWR, Boroda S, Nian W, Levental KR, Levental I, Harris TE, Chang JT, Du G: Lipin-1 regulation of phospholipid synthesis maintains endoplasmic reticulum homeostasis and is critical for triple-negative breast cancer cell survival. FASEB J 2017;31:2893-2904.

26 El-Serag HB: Hepatocellular carcinoma. New Engl J Med 2011;365:1118-1127.

27 Kamerkar S, LeBleu VS, Sugimoto H, Yang S, Ruivo CF, Melo SA, Lee JJ, Kalluri R: Exosomes facilitate therapeutic targeting of oncogenic KRAS in pancreatic cancer. Nature 2017;546:498-503.

28 Takahasi K, Iinuma H, Wada K, Minezaki S, Kawamura S, Kainuma M, Ikede Y, Shibuya M, Miura F, Sano K: Usefulness of exosome-encapsulated microRNA-451a as a minimally invasive biomarker for prediction of recurrence and prognosis in pancreatic ductal adenocarcinoma. J Hepatobiliary Pancreat Sci 2017;25:155161.

29 Latchana N, Abrams ZB, Howard JH, Regan K, Jacob N, Fadda P, Terando A, Markowitz J, Agnese D, Payne P, Carson WE, 3rd: Plasma microRNA levels following resection of metastatic melanoma. Bioinform Biol Insights 2017;11:1177932217694837.

30 Chen Q Hu H, Jiao D, Yan J, Xu W, Tang X, Chen J, Wang J: miR-126-3p and miR-451a correlate with clinicopathological features of lung adenocarcinoma: The underlying molecular mechanisms. Oncol Rep 2016;36:909-917.

31 Liu ZR, Song Y, Wan LH, Zhang YY, Zhou LM: Over-expression of miR-451a can enhance the sensitivity of breast cancer cells to tamoxifen by regulating 14-3-3zeta, estrogen receptor $\alpha$, and autophagy. Life Sci 2016;149:104-113. 
Cellular Physiology Cell Physiol Biochem 2019;53:19-35

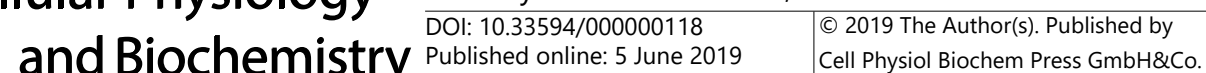

\begin{tabular}{l|l} 
Published online: 5 June 2019 & Cell Physiol Biochem Press Gmb \\
\hline Zhao et al.: Exosomal miR-451a Targets LPIN1 to Suppress HCC
\end{tabular}

32 Li HP, Zeng XC, Zhang B, Long JT, Zhou B, Tan GS, Zeng WX, Chen W, Yang JY: miR-451 inhibits cell proliferation in human hepatocellular carcinoma through direct suppression of IKK-beta. Carcinogenesis 2013;34:2443-2451.

33 Minna E, Romeo P, Dugo M, De Cecco L, Todoerti K, Pilotti S, Perrone F, Seregni E, Agnelli L, Neri A, Greco A, Borrello MG: miR-451a is underexpressed and targets AKT/mTOR pathway in papillary thyroid carcinoma. Oncotarget 2016;7:12731-12747.

34 Brohee L, Demine S, Willems J, Arnould T, Colige AC, Deroanne CF: Lipin-1 regulates cancer cell phenotype and is a potential target to potentiate rapamycin treatment. Oncotarget 2015;6:11264-11280. 